\title{
Schizotypal traits and N400 in healthy subjects
}

\author{
MARIE PRÉVOST, ${ }^{\mathrm{a}, \mathrm{b}}$ MITCHELL RODIER,,${ }^{\mathrm{c}}$ LOUIS RENOULT,${ }^{\mathrm{a}, \mathrm{b}}$ YVONNE KWANN, ${ }^{\mathrm{a}}$ \\ EMMANUELLE DIONNE-DOSTIE, ${ }^{\mathrm{a}}$ ISABELLE CHAPLEAU, ${ }^{\mathrm{a}}$ MATHIEU BRODEUR, ${ }^{\mathrm{a}, \mathrm{c}}$ \\ CLAIRE LIONNET, ${ }^{\mathrm{a}}$ AND J. BRUNO DEBRUILLE ${ }^{\mathrm{a}, \mathrm{b}, \mathrm{c}}$ \\ ${ }^{a}$ Douglas Mental Health University Institute, Montréal, Québec, Canada \\ ${ }^{\mathrm{b}}$ Department of Neurology and Neurosurgery, McGill University, Montréal, Québec, Canada \\ ${ }^{\mathrm{c}}$ Department of Psychiatry, McGill University, Montréal, Québec, Canada
}

\begin{abstract}
We examined whether correlations previously found between symptoms of schizophrenia patients and the amplitude of an event-related potential (ERP), the N400, could be also found between schizotypal experiences of healthy subjects and the N400. We chose a semantic categorization task previously used with patients. Schizotypal experiences were measured with the schizotypal personality questionnaire (SPQ). The effects of the other factors were controlled for when assessing the correlations between each SPQ factor and N400s. These correlations were assessed at each electrode site to see whether their distribution on the scalp follows that of the N400 effect. Disorganization and interpersonal scores were found to correlate with ERPs in the N400 time window, as previously reported for the comparable symptoms of patients. However, the scalp distribution of these correlations differed from that of the N400 effect.
\end{abstract}

Descriptors: Delusional ideation, Disorganization, Interpersonal factor, Event-related brain potentials, N400

Research into the symptoms of schizophrenia focuses primarily on the three dimensions that are frequently used to describe schizophrenia, which are reality distortion, disorganization, and negative symptoms (Lenzenweger, 1999). Interestingly, experiences which resemble these symptoms can be observed in the general population (Raine, 1991; Reynolds, Raine, Mellingen, Venables, \& Mednick, 2000; Verdoux \& van Os, 2002). This finding has raised the idea that, rather than a dichotomy, there could be a continuum between schizophrenia and normality (Claridge, 1997; Johns \& van Os, 2001; Shevlin, Murphy, Dorahy, \& Adamson, 2007; Strauss, 1969). Although the dichotomous view of psychopathology remains central to mental health care and research, there is a growing debate between these two approaches. One way to go forward is to test whether symptoms of schizophrenia patients and schizotypal personality traits of normals are associated with similar cognitive anomalies, since, as shown by Johns and van Os (2001), such similarities would reinforce the notion of a continuum. An increasing number of studies have looked at the relationship between schizotypal traits in healthy participants and cognitive functions. They observed in these participants similar cognitive anomalies as those found in schizophrenia patients (e.g., Bed-

We thank the subjects, whose participation was essential in the completion of this study. Funding was provided by Fonds de la Recherche en Santé Québec (scholarship 10084 to J. B. D.; fellowship 13542 to L. R.) and National Alliance for Research on Schizophrenia and Depression (NARSAD Young Investigator Award to J. B. D.).

Address reprint requests to: Dr. J. Bruno Debruille, Human Neurocognitive Science Laboratory, F. B. C. Pavilion, Douglas Mental Health University Institute, 6875 Blvd. LaSalle, Montréal, (QC) H4H 1R3, Canada. E-mail: bruno.debruille@douglas.mcgill.ca well, Kamath, \& Compton, 2009; Johnston, Rossell, \& Gleeson, 2008; Wilson, Christensen, King, Li, \& Zelazo, 2008).

Semantic processes are among the cognitive processes that have been widely studied in schizophrenia. The N400, an eventrelated potential (ERP) evoked by any potentially meaningful stimulus and thought to reflect semantic processing, has been related to reality distortion, disorganization, and negative symptoms of schizophrenia patients in various studies (e.g., Andrews, Shelley, Ward, Fox, Catts, \& McConaghy, 1993; Debruille, Kumar, Saheb, Chintoh, Gharghi, et al., 2007; Kostova, Passerieux, Laurent, \& Hardy-Baylé, 2005, respectively). In the next paragraphs, these three relations will be reviewed.

The first relation, that between reality distortion and the N400, has been studied because of the ostensible tendency of patients to maintain their delusions in the face of disconfirmatory evidence. It has been hypothesized that this 'tendency' is related to a deficit in integrating the information that challenges their beliefs (Freeman, 2007; Moritz \& Woodward, 2006). The N400 was thus used as an index of this integration since the amplitude of this ERP appears to be proportional to the amount of effort that is spontaneously deployed to integrate unexpected semantic information into cognitive representations (e.g., Holcomb, 1993). Stimuli that arguably trigger more integration effort, such as stimuli that do not match the context, elicit N400s of greater amplitudes than stimuli that are primed because they match preceding stimuli. Consistent with the N400 integration hypothesis and with their deficit in integrating disconfirmatory evidence, schizophrenia patients who were more delusional were found to have smaller N400s than patients who were less delusional (Debruille et al., 2007). The link between N400 and delusion severity in schizophrenia patients was confirmed by the 
results of Kiang, Kutas, Light, and Braff (2007). These authors reported that the severity of psychotic symptoms (i.e., delusions and hallucinations) correlates with a reduction of the N400 effect, that is, with a reduction of the difference between the amplitude of the N400 to mismatching stimuli and that to matching stimuli. Since the variations of one or of both of these amplitudes could be responsible for smaller N400 effects, one of the aims of the present study was to carefully differentiate the three N400 measures. In the rest of this paper, 'N400 amplitude' will be used only to designate the raw amplitude of the N400 deflections, whereas the phrase 'N400 effect' will be exclusively devoted to N400 differences.

The second relation, that between disorganization and N400, has been studied partly because of the deficit found in schizophrenia patients in processing context efficiently and in keeping it in working memory during long stimulus onset asynchronies (SOAs) (Cohen \& Servan-Schreiber, 1992; Hardy-Baylé, Sarfati, $\&$ Passerieux, 2003; Hemsley, 2005). Accordingly, a word that is related to a following target word induces less priming in patients than in normal controls. One should thus observe a correlation in the direction opposite to that predicted for delusions. N400s elicited by primed target words should be larger in more- than in less-disorganized patients (see Discussion section for another hypothesis leading to the same prediction). The results of previous works support that prediction. Correlations were found between thought disorder and larger N400 amplitudes for congruent targets in Kuperberg, Sitnikova, Goff, and Holcomb (2006) and in Kostova et al. (2005, personal communication), which possibly explain the smaller N400 effects observed in Ditman and Kuperberg (2007). Interestingly, one study described greater N400s for the incongruent targets for higher thought disorder scores (Salisbury, O'Donnell, McCarley, Nestor, \& Shenton, 2000), while another mentioned greater amplitudes for the mean of congruent targets and incongruent targets (Andrews et al., 1993). It has to be noted that in all the above mentioned studies, the word or the sentence used to prime the target stimulus was shown just once in the experiment. This can only increase the impact of the problematic maintenance of this context information in working memory and further jeopardize the facilitation this context induces and account for the larger N400 amplitudes obtained for the targets.

However, correlations between N400s and the two symptoms mentioned above were not always observed (Ditman \& Kuperberg, 2007; Kostova et al., 2005; Salisbury et al., 2000). For delusion, one study reported an opposite relationship, that is, greater N400 amplitudes with higher delusion scores (Kiang, Kutas, Light, \& Braff, 2008). For disorganization, the correlations with N400s sometimes failed to be found (Kiang et al., 2007, 2008), and one study described a relationship going in the reverse direction, that is, reduced N400 amplitudes for stimuli that do not match the context (non-exemplar category) for highly disorganized patients (Debruille et al., 2007). Nevertheless, these discrepancies may not be so surprising. Because delusions and disorganization can be positively correlated to each other (Lenzenweger \& Dworkin, 1996; for comparable experiences in the general population, see Raine, Reynolds, Lencz, Scerbo, Triphon, \& Kim, 1994; Reynolds et al., 2000) their opposite influences on N400 amplitudes could cancel each other, or one could even reverse the influence of the other. Therefore, in the present study, which aimed at evaluating the relationship between schizotypal traits in healthy participants and the N400, it was decided to use partial correlations in order to assess the effect of one schizotypal trait on N400s while controlling for the effects of the two other traits.

Similarly, the third relation, that between the negative symptoms of schizophrenia and the N400, was not found in some studies (Ditman \& Kuperberg, 2007; Kiang et al., 2008; Kuperberg et al., 2006; Salisbury et al., 2000). But two studies with schizophrenia patients found a tendency for a reduced N400 effect with more negative symptoms (Kiang et al., 2007; Kostova et al., 2005). Moreover, when looking at similar schizotypal traits in healthy subjects, one study reported significant results. Kiang and Kutas (2005) observed a correlation between diminished N400 effects and higher scores for the interpersonal items of the Schizoptypal Personality Questionnaire, the SPQ (Raine, 1991), which reflect experiences that resemble negative symptoms in patients. As this interpersonal factor also includes paranoid ideation and social anxiety, the authors proposed that a mistrust bias might be responsible for this finding. They based their proposition on a study where mistrust was induced in university students (Schul, Mayo, \& Burnstein, 2004). In this study, behavioral responses showed both an increased semantic priming effect for words having a meaning opposite to that of the prime (e.g., hollow-full) and a decreased effect for words that were synonyms (e.g., hollow-empty). The authors of this behavioral study proposed that "mistrust may be associated with increased activation of message-incongruent associations and decreased activation of message-congruent associations, by encouraging a person to focus more on the possibility that a message is invalid." Kiang and Kutas (2005) thus related their smaller N400 effects to this decreased activation of message-congruent associations.

In addition to the interpersonal score, the SPQ can also be used to measure the disorganization trait and delusional-like ideation in healthy subjects. The idea that these experiences of healthy subjects resemble schizophrenia symptoms has been supported in several studies (Raine, 1991; Reynolds et al., 2000; Verdoux \& van Os, 2002; for experiences which resemble delusions, see Peters, Joseph, \& Garety, 1999; and van Os, Hanssen, Bijl, \& Ravelli 2000; for traits which resemble disorganization, see Coleman, Levy, Lenzenweger, \& Holzman, 1996; and Gooding, Tallent, \& Hegyi, 2001; for traits that resemble negative symptoms, see Chapman, Edell, \& Chapman, 1980; and Cadenhead, Kumar, \& Braff, 1996). Nevertheless, to stress the fact that there may be qualitative and quantitative differences between the symptoms of patients and schizotypal traits, a different terminology is used for healthy subjects. The phrase 'disorganization trait' is used to refer to experiences that resemble disorganization and the phrase 'delusional-like ideation' is used to refer to experiences evaluated by the SPQ that are observed in healthy subjects and that resemble delusions in patients.

Recent studies using schizotypal personality measures have demonstrated that ERPs may be used to study neurocognitive processes related to schizotypal traits in healthy individuals (Gassab, Mechri, Dogui, Gaha, d'Amato, et al., 2006; Kiang \& Kutas, 2005; Wan, Crawford, \& Boutros, 2006; Wang, Miyazato, Hokama, Hiramatsu, \& Kondo, 2004). Although to a lesser extent than in the case of schizotypal personality disorders, the existence of measurable schizotypal traits in healthy populations may be seen as an opportunity for approaching the mechanisms of schizophrenia symptoms in a less confounded context. Indeed, whereas some patients' anomalies might also be the consequences of the long-term disability induced by symptoms, this is less likely to be the case in healthy populations. Moreover, as with schizotypal personality disorders, studying 
healthy subjects allows interpretations free from the problems of patients' medication.

The aim of the present study was thus to explore the neurocognitive mechanisms underlying schizotypal personality traits in healthy individuals. More specifically, our goal was to see whether we could find in these subjects the relationships observed in patients between N400 and the three schizophrenia symptoms, namely, the smaller N400s with higher delusions scores, the greater N400s with more disorganization, and the smaller N400 effects in case of more severe negative symptoms. Although acknowledging that schizotypal personality traits of healthy subjects may be quantitatively and qualitatively different from schizophrenia symptoms, we aimed at testing whether these traits are accompanied by cognitive processes similar to those of patients, which would bring further support to the idea of a continuum between schizophrenia and normality (Peters et al., 1999; van Os et al., 2000).

In addition to the above-mentioned use of partial correlations to control for the effect of the other schizotypal factors when focusing on the relation of one of them with the N400, three features characterized the present study. First, we used the protocol of our previous study in patients (Debruille et al., 2007). This was done both to compare results obtained in patients to those in normals and to circumvent the following interpretation problem. In studies where each target word was preceded by a particular context word, the anomalous N400s to targets observed were seen as due to an abnormal priming of targets. This abnormality was thought to be related to a deficient processing of the context or to a problematic maintenance of the prime in working memory. However, if the processing of context words is impaired, the processing of targets also may be impaired and nothing can help to determine which of the two possibilities accounts for the anomalous target N400s. In the semantic categorization task of Debruille et al. (2007), the same category word was used as a context (i.e., first word of the trial) for all the trials for which participants had to make a decision. This was aimed at facilitating the processing of the context so that it produced the same effects on the processing of the target word (i.e., on the second word of the trial) in patients as in normals. A previous study using this particular protocol reported an absence of significant differences between patients and normal controls in the ERPs evoked by such invariant context words (Debruille, Kumar, Saheb, Chintoh, Gharghi, et al., 2010). Thus, if the disorganization trait is found to have an effect on the $\mathrm{N} 400$ s to the targets of our study, it will be more likely to be due to a deficit in processing the targets themselves, which would, nevertheless, have to be confirmed by an absence of effects of schizotypal traits on each of the components of the ERPs elicited by the context word.

The second feature that characterizes the present study is to compute the correlations for each electrode site. For written words, the N400 effect reaches a maximum over midline centroparietal electrodes and is slightly larger over right than left parietal sites (Holcomb, 1988; Kutas, van Petten, \& Besson, 1988). Correlations involving the N400 effect can thus be expected to reach a maximum at these sites.

The third feature of this study derives from the fact that variations in the size of the N400 effects may have several causes. A smaller N400 effect can be due to larger N400 deflections for matching stimuli, to smaller N400 deflections for mismatching stimuli, or to both. As mentioned above, when discussing the larger N400 deflections found with disorganization and with the interpersonal factor in the matching conditions, such results have different functional significances. Therefore, correlations for each of the three schizotypal factors were measured three times, once with the N400s elicited by matching stimuli, once with the $\mathrm{N} 400$ s for mismatching stimuli, and once for the N400 effects.

\section{Methods}

\section{Participants}

Forty-nine right-handed participants ( 35 women, 14 men) aged between 18 and 51 years were recruited by advertisements in an English and in a French newspaper. Participants who answered these ads were asked what their mother tongue was. Only English and French were accepted. The rest of the procedure was carried out in the language of the participants. They had to have normal or corrected-to-normal vision and were screened by telephone and systematically excluded for any history of DSM-IV Axis I psychiatric illnesses, except for depressive episodes that resolved at least 2 years ago. The use of these a priori criteria led to include 2 participants who had suffered from depression. Both had recovered and were off medication for more than 6 years. Participants with a history of head injury with loss of consciousness longer than 10 min were excluded, as well as participants with neurological or medical conditions known to compromise brain function and participants abusing drugs. The procedure to recruit the participants included first a short questionnaire made up of the 16 items of the 'odd belief and magical thinking' and 'ideas of reference' subscales of the SPQ (Dumas, Bouafia, Gutknecht, Saoud, Dalery, \& d'Amato, 2000; Dumas, Rosenfeld, Saoud, Dalery, \& d'Amato, 1999; Raine, 1991). These were used to preassess delusional-like ideation over the phone. In the first phase of the recruitment, only those who scored 5 or more out of 16 (the maximal score) were asked to participate in the study $(n=25)$ in order to have enough participants with high delusional-like ideation scores. No or low delusional-like ideation participants $(n=24)$ were recruited in a second phase among people with delusional-like ideation scores smaller than 5 and whose demographic characteristics (age, gender, and number of years of education) matched those of the high delusional-like ideation score participants. At their arrival in the lab, participants gave written informed consent after the procedures were described according to the criteria of the Research Ethics Board of the Douglas Mental Health University Institute.

Before having their ERPs recorded, participants had to complete the SPQ. This questionnaire is based on the DSM-III-R criteria for schizotypal personality disorder and includes nine subscales that can be grouped into three clusters or factors: (1) interpersonal, (2) disorganization, and (3) cognitive-perceptual (Raine et al., 1994). The validity of the whole SPQ has been demonstrated (Raine, 1991). Its clusters have been defined by a factor analysis (Raine et al., 1994) and used in many previous studies (e.g., Dickey et al., 2005; Kiang \& Kutas, 2005; Sommer, Daalman, Rietkerk, Diederen, Bakker, et al., 2008). The interpersonal score is computed by adding the scores for the 'social anxiety,' the 'no close friends,' the 'constricted affect,' and the 'paranoid ideation' subscale. The disorganization trait score is computed by adding the score for the 'odd speech' subscale to the score for the 'odd or eccentric behavior' subscale. Both subscales load on the same factor (disorganization) in the general population and in clinical population (Reynolds et al., 2000). The 'cognitive and perceptual' cluster could not be used to evaluate 
Table 1. Characteristics of the Participants ( $1^{\text {st }}$ column) and Correlations (Pearson's $r$ Coefficients) between these Characteristics (following columns)

\begin{tabular}{|c|c|c|c|c|c|c|}
\hline & Mean (Stdev) & Age & Years of study & SPQ & Interpersonal & Disorganization \\
\hline Age & $30.3(10.4)$ & & & & & \\
\hline Years of study & $14.3(1.7)$ & 0.00 & & & & \\
\hline SPQ & $18.9(14.1)$ & $-0.31^{*}$ & -0.13 & & & \\
\hline Interpersonal & $7.2(6.2)$ & $-0.30^{*}$ & -0.15 & $0.90^{* * * * *}$ & & \\
\hline Disorganization & $4.5(3.7)$ & $-0.34^{*}$ & -0.17 & $0.85^{* * * *}$ & $0.68 * * * *$ & \\
\hline Delusion & $5.1(4.2)$ & -0.22 & -0.04 & $0.85^{* * * *}$ & $0.62 * * * *$ & $0.62 * * * *$ \\
\hline
\end{tabular}

Note: SPQ: Total score for the schizotypal personality questionnaire.

$* p<0.05 ; * * * * 0.001$.

delusional-like ideation since it also includes hallucinations. It is thus the scores for the two subscales 'idea of references' and 'odd beliefs and magical thinking' that were used here, as previously done in a study performed to assess the neurocognitive mechanisms underlying delusional-like ideation in normals (e.g., Woodward, Buchy, Mortiz, \& Liotti, 2007). Here, these two scores were simply added. Note that paranoid ideation was not integrated into the delusional-like ideation score because this subscale does not include delusional-like items per se, but rather suspiciousness, which is already included in the interpersonal factor.

Pearson correlations between subjects' characteristics are displayed in Table 1. Each SPQ factor correlated significantly with the two others. Additionally, age negatively correlated with the overall SPQ scores and with the interpersonal and disorganization factors but not with delusional-like ideation.

\section{Task}

Subjects were seated comfortably in a dimly lit room in front of a computer screen placed $1 \mathrm{~m}$ from their eyes. Black stimuli were presented on a white background at the center of this screen. Trials were made of two serially presented words. In two-thirds of the trials, the first word was the question word 'ANIMAL?' and, in one-third of the trials, the first word was the instruction 'INACTION.' These words were then followed by the target word. The stimulus onset asynchrony was $2 \mathrm{sec}$ and each word was displayed for $1 \mathrm{sec}$. The target word was either an exemplar of the animal category (e.g., lion) or a non-exemplar of this category (e.g., hammer). Subjects had to decide whether or not the target word belongs to the animal category as rapidly and as accurately as possible by pressing one of two keys with their right index finger. The 'INACTION' word, introduced so that subjects have to pay attention to the first word, signaled to the participants that they should not respond to the target stimulus, which was also either an exemplar or a non-exemplar of the animal category. The non-exemplar stimuli comprised names of tools, pieces of furniture, office and kitchen objects, buildings and parts of buildings, and transportations means. The target word was followed 2 to $2.5 \mathrm{sec}$ later by the word 'Blink,' giving the subjects the opportunity to blink without disrupting the electroencephalogram (EEG) signal of the trial. All target words used were selected from among familiar words using the Content, Mousty, and Radeau's database (1990) for the French words and the Kucera and Francis (1967) counts for the English words. In the French version, in both the exemplar and the non-exemplar categories, there were forty-seven words with a frequency comprised between 0 and 1000 out of 100 million occurrences, nine words with a frequency comprised between 1000 and 3000, and four words with a frequency higher than 3000. In the English version, in both categories, there were forty-seven words with a frequency comprised between 0 and 9 out of one million; ten words with a frequency comprised between 10 and 17, and three words with a frequency comprised between 35 and 117 . The average length of words in each category did not differ from one another. In the French version, the mean number of letters was $6.5 \pm 1.7$ in the exemplar category and $6.7 \pm 1.5$ in the non-exemplar category. In the English version, the mean number of letters was $5.5 \pm 1.8$ in the exemplar category and $5.75 \pm 1.7$ in the non-exemplar category. In the action condition, there were 60 trials with exemplar target words and 60 trials with non-exemplar target words. For the 'INACTION' condition, there were 30 trials for each of the two stimulus categories. So, for both conditions, the probability of occurrence of an animal target word was $50 \%$. Each target word was presented only once, thus the probability of occurrence of a particular target word was 1 out of 180 .

\section{Data Acquisition}

Behavioral responses were recorded for each trial. The EEG was recorded with tin electrodes from the ECI cap (Electro-Cap International, Eaton, $\mathrm{OH}$ ), which were placed according to the modified expanded 10-20 system (American EEG Society, 1991) with a right ear lobe initial reference. Twenty-eight electrodes were used. Sites were grouped into three subsets. The sagittal subset included $\mathrm{Fz}, \mathrm{FCz}, \mathrm{Cz}$, and $\mathrm{Pz}$; the parasagittal subset, $\mathrm{FP} 1 / 2, \mathrm{~F} 3 / 4, \mathrm{FC} 3 / 4, \mathrm{C} 3 / 4, \mathrm{CP} 3 / 4, \mathrm{P} 3 / 4, \mathrm{O} 1$, and $\mathrm{O} 2$; and the lateral subset, F7/8, FT7/8, T3/4, TP7/8, T5, and T6. Eye movements and blinks were monitored with F7 and F8 for horizontal movements and with FP2, and an additional electrode placed on the right cheekbone for vertical movements. The impedance was kept below $5 \mathrm{~K} \Omega$. The gain of the Contact Precision Instruments amplifiers (Boston, MA) used was set at 20,000. The half amplitude cut-offs of high and low pass frequency filters were set at 0.01 and $100 \mathrm{~Hz}$, respectively. In addition, a $60-\mathrm{Hz}$ electronic notch filter was used. EEG signals were digitized at a $256-\mathrm{Hz}$ sampling frequency ${ }^{1}$ and stored along with the stimulus and response codes. The EEG was re-referenced off-line to the mean of the left and right earlobes signals.

\section{Measures}

EEG epochs corresponding to trials with incorrect responses or with reaction times shorter than $200 \mathrm{~ms}$ or longer than $2000 \mathrm{~ms}$

\footnotetext{
${ }^{1}$ The 256-Hz sampling frequency respects Nyquist's law, but does not eliminate the possibility of signal distortion in some cases.
} 
were rejected. We also rejected trials with excessive eye movements, amplifier saturations, or analog-to-digital clippings lasting more than $100 \mathrm{~ms}$. Our baseline was set between -200 and 0 $\mathrm{ms}$. Averages were calculated over the time period between target word onset and $1000 \mathrm{~ms}$ later. The minimum numbers of artifactfree trials averaged by subject was 28 and 30 for the exemplar and the non-exemplar categories, respectively. The mean number of trials averaged was 49 (SD 8) in both the exemplar category and the non-exemplar category. The N400 amplitude was measured by computing the mean voltage of the ERPs in a 350-550 ms time window, relatively to the baseline. This time window was centered on the negative peak observed on the grand average of the non-exemplar-exemplar ERP subtractions. For each subject, a mean voltage was computed at each electrode for the N400 in the exemplar category, for the N400 in the non-exemplar category, and for the N400 difference (non-exemplar minus exemplar amplitudes).

\section{Analyses}

Mean reaction times (RTs) and accuracies (As) were obtained in the action condition, that is, for all trials except those with INACTION as the prime word. Two subjects were excluded from all analyses because their error rate was greater than $10 \%$. RTs and As were analyzed with separate one-way repeated-measure analysis of variance (ANOVAs) (i.e., $t$-tests) with category (exemplar versus non-exemplar) as the within-subject factor. Assuming a linear decomposition, partial correlations were run to control for the effect of the two other schizotypal factors. These correlations were run between each of the three factors and RTs on the one hand, and each of the three factors and As on the other hand. This was done in an exploratory way to generate a priori hypotheses for future studies.

For mean voltages of ERPs in the N400 time window, a twoways repeated measure omnibus ANOVA was run with category (exemplar vs. non-exemplar) and electrode site as within-subjects factors. The three subsets of electrodes were analyzed separately when an interaction between the category factor and the electrode was observed in the omnibus ANOVA. For the parasagittal and lateral subsets of electrodes, the hemi-scalp (right versus left) was added as a third within-subject factor. We used the Greenhouse and Geisser (1959) procedure to compensate for heterogeneous variances for the factor having more than two levels, that is, the electrode factor. In each case, the original degrees of freedom are reported together with the Epsilon (E) correction factor and the corrected probability level.

The mean voltage values of the N400s in the exemplar category, the N400s in the non-exemplar category, and the N400 effect (non-exemplar minus exemplar differences) were correlated (two-tailed) to the delusional-like ideation, the interpersonal and the disorganization factor scores at each electrode, to assess the scalp distribution of the effects. Partial correlations between ERP measures at each electrode and each SPQ subscale scores were then computed. This allowed controlling for the two remaining SPQ factors scores, which was necessary since significant correlations were found between these factors (see Table 1) and since their comparable symptoms have been found to have opposite effects on ERPs in the N400 time window in studies with schizophrenia patients (e.g., Andrews et al., 1993; Kuperberg et al., 2006). Partial correlations were possible since correlations could be assumed to be linear. For the correlation between disorganization trait and interpersonal, the Pearson's $r$ was 0.68 (see Table 1). The use of a 6 degrees polynomial function to find the best fit only improves the $r$ to 0.71 . The initial straight line was thus an adequate solution. For the correlation between the disorganization trait and delusional-like ideation, the Pearson's $r$ was 0.62 . The use of the same type of complex function only improves the $r$ to 0.64 . Finally, for the correlation between delusional-like ideation and the interpersonal trait, for which the Pearson's $r$ was 0.62 , the use of a complex polynomial function only improves the $r$ to 0.64 . Pearson, and thus linear correlation, was therefore a good model to represent the association between factors.

\section{Results}

\section{Behavioral Data}

Mean RTs were shorter in the exemplar $(843 \mathrm{~ms} \pm 122)$ than in the non-exemplar $(923 \mathrm{~ms} \pm 160)$ category $(F(1,45)=55.4$, $p<.001)$. Accuracies (i.e., error rates) did not differ significantly between the exemplar $(2.8 \% \pm 3.5)$ and the non-exemplar $(2.3 \% \pm 3.1)$ categories. No correlation with delusional-like ideation, disorganization trait, or interpersonal scores was found, either with RTs or with error rates, even when running partial correlations and thus when studying the effect of one factor while controlling for the two others.

\section{ERP Data}

ERPs in the N400 time window were more negative in the nonexemplar than in the exemplar category as showed by the omnibus ANOVA $(F(1,45)=53.6, p<.001)$. Since the category factor interacted with electrode $(F(27,1215)=6.5, \mathrm{E}=0.169$, $p<.001$ ), we conducted ANOVAs for each subset of electrodes and observed that ERP amplitudes in the non-exemplar category were greater than in the exemplar category for the three subsets (sagittal: $F(1,46)=45.2, p<.001$, parasagittal: $F(1,45)=51.5$, $p<.001$, and lateral: $F(1,46)=46.5, p<.001)$, as observed in Figure 1. ERPs were more negative over the left than over the right hemi-scalp at the parasagittal $(F(1,45)=25.2 ; p<.001)$ and lateral $(F(1,46)=13.1, p<.001)$ subsets (Figure 2$)$. In contrast, a category $\times$ hemi-scalp interaction at the parasagittal subset $(F(1,45)=4.2, p=.045)$ revealed that the difference between the exemplar and the non-exemplar categories was larger over the right than over the left side of the scalp (Figures $3 \& 4$ ). The post hoc analyses performed showed that this difference was significant for each hemi-scalp at the parasagittal subset (left: $F(1,45)=44.9, p<.001$; right: $F(1,45)=53.8, p<.001)$.

The Pearson correlations between each SPQ factor and ERP amplitudes in the N400 time window were assessed for each of the 28 electrodes. Partial correlations were then systematically computed to control for the two other SPQ factors. As displayed in Figure 5, no significant bivariate correlation between delusional-like ideation and ERP amplitudes was found over centroparietal electrodes. Some bivariate correlations were observed at frontal electrode sites that were no longer significant when we controlled for the interpersonal and the disorganization scores.

The disorganization scores negatively correlated with ERP voltages in the N400 time window over all the frontal and central electrodes in the exemplar and non-exemplar categories. The higher the disorganization scores, the more negative the ERPs were in the N400 time window at these sites in both categories. These correlations remained significant after adjustment for delusional-like ideation and interpersonal scores, as shown by Figure 5. However, the N400 effect did not correlate with the disorganization factor, which suggests that the increase in 


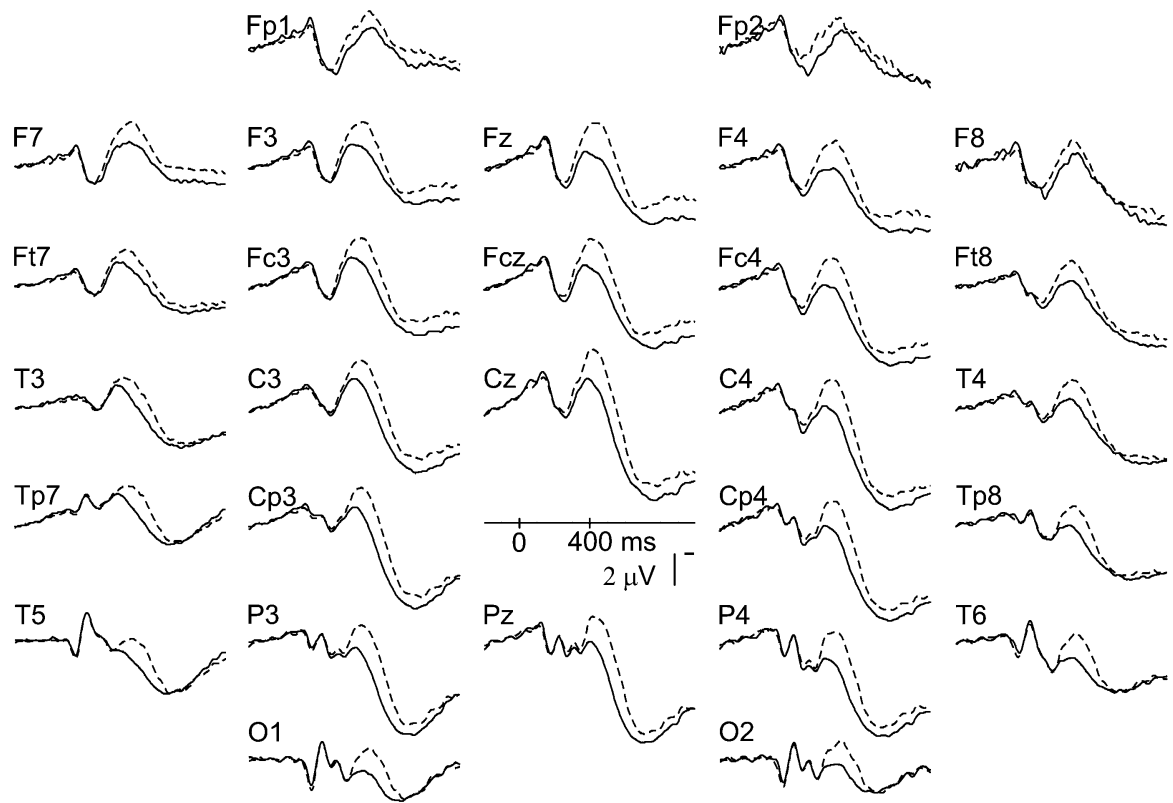

Figure 1. Grand average ERPs for the exemplar and the non-exemplar categories $(n=47)$.

negativity induced by the disorganization trait was similar in the exemplar and the non-exemplar category.

Regarding the interpersonal factor, no correlation was found with the ERP amplitude in the exemplar and non-exemplar categories after adjustment for delusional-like ideation and disorganization scores (Figure 5). In contrast, there were positive correlations between the N400 effect and interpersonal scores, which remained significant over the left central electrodes when delusional-like ideation and disorganization scores were controlled for. Smaller N400 differences were associated with higher interpersonal scores.

As age correlated with disorganization and interpersonal factors, each partial correlation was also run controlling for age in addition to the other factors. Correlations were also run without the two participants with a history of depression. This did not change the correlations found or their scalp distribution.

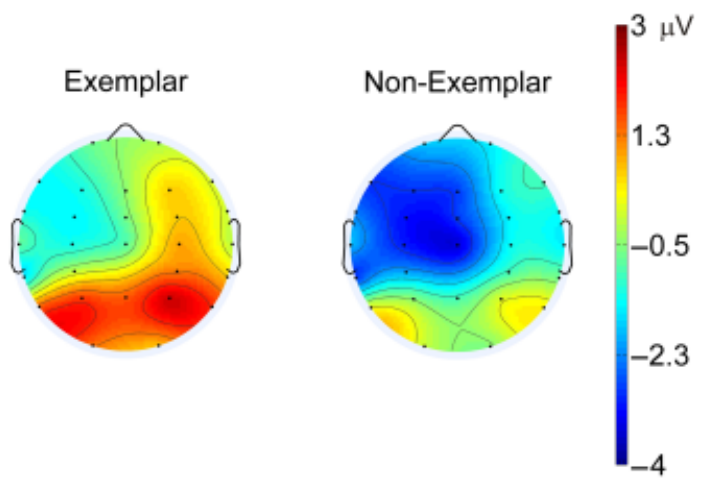

Figure 2. Spline interpolated voltage maps of the N400 amplitude for the exemplar and the non-exemplar categories in the $350-550 \mathrm{~ms}$ time window.

\section{Discussion}

We investigated in healthy subjects the relationship between delusional-like ideation, disorganization trait, and interpersonal factors, and the N400 potential elicited during a semantic categorization task. As sometimes observed in patients (Debruille et al., 2007; Lenzenweger \& Dworkin, 1996), and often in healthy populations (Raine et al., 1994; Reynolds et al., 2000), the scores for each factor were significantly correlated with the scores for the two other factors. Individuals who had higher delusion-like ideation scores also tended to have higher disorganization scores and greater scores for the interpersonal factor (see Table 1). The effect of each schizotypal factor was thus studied while controlling for the effects of the two remaining factors. The N400 protocol used was designed to circumvent the effect of the deficit of

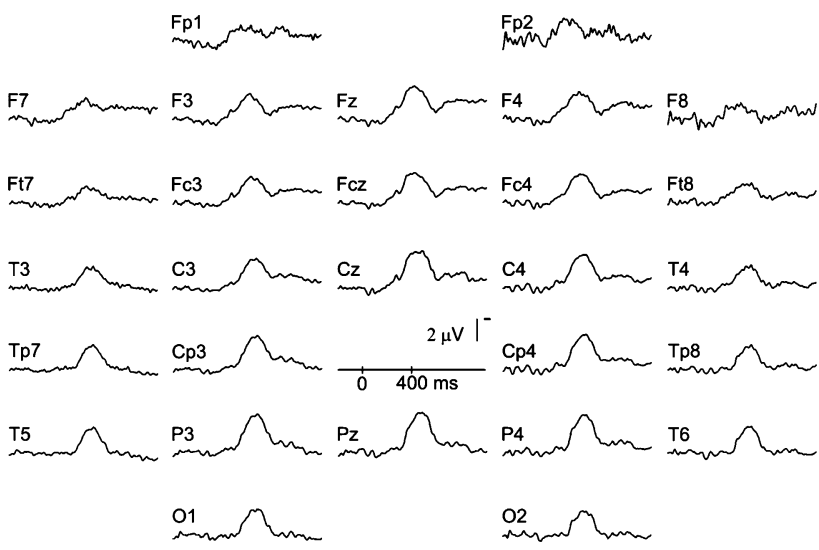

Figure 3. Grand average ERPs of the N400 effect (non-exemplar minus exemplar ERPs) $(n=47)$. 


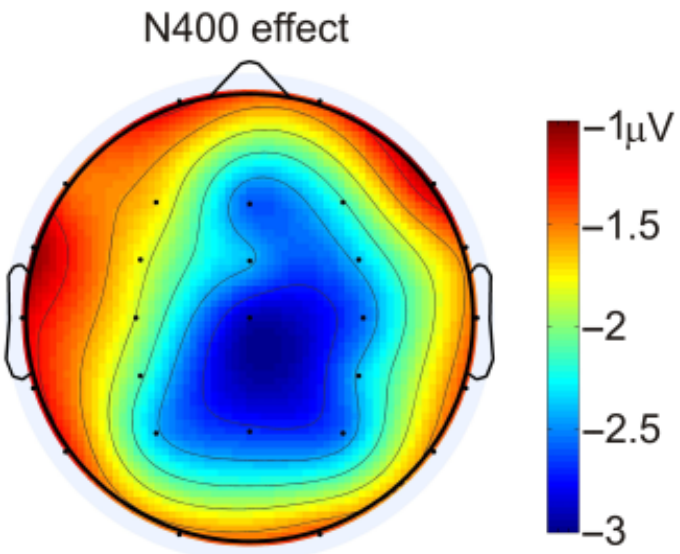

Figure 4. Spline interpolated voltage maps of the N400 effect (nonexemplar minus exemplar ERPs) in the 350-550 ms time window.

the processing of prior context (Debruille et al., 2010). The source of the variations of N400 effects was assessed by measuring correlations in both the exemplar and the non-exemplar categories. Finally, the correlations were computed at each electrode site, to test whether the relationship under investigation follows the scalp distribution of the N400 effect obtained in the experiment between exemplar and non-exemplar targets. The results obtained demonstrated the relevance of this method. Correlations between these ERPs and disorganization and interpersonal factors were observed, but their scalp topography did not follow that of the N400 effect. The few correlations found between ERPs in the N400 time window and delusion-like ideation became insignificant when controlling for the two other factors (Figure 5).

The N400 protocol used herein led to a negative deflection in the N400 time window for both categories. This deflection was widespread over the scalp. The N400 in the non-exemplar category was significantly greater than the N400 in the exemplar category, and this ERP difference had a centro-parietal maximum greater over the right than over the left hemiscalp (Figure 4). These results suggest that our protocol, where a single category is shown as a first word, elicits the classical N400 effect found for written words (Holcomb, 1988; Kutas \& Federmeier, 2000).

Significant correlations between N400 amplitudes and disorganization scores were found over centro-parietal electrode sites. This replicates the results of previous studies done with patients (Andrews et al., 1993; Kostova et al., 2005; Kuperberg et al., 2006; Salisbury et al., 2000). The more disorganized the subjects, the larger their N400 deflections. In the present study, this was significant not only in the exemplar but also in the non-exemplar category. The correlations remained significant after controlling for the two other factors. Interestingly, no correlation with the N400 effect was observed, as in Kiang and Kutas' study (2005) with healthy subjects. In both studies, the disorganization trait may have enhanced N400 amplitudes similarly in the exemplar and non-exemplar categories, leaving the N400 effect unchanged. The fact that this was obtained here in a task created to circumvent the effect of the deficit in the processing of the prior context suggests that larger target N400s could be caused by an anomalous semantic processing of target stimuli themselves. Such anomalous processing could have also existed in previous studies, in addition to the deficiency in the processing of the context that was suggested. Most interestingly, while part of the correlations were observed at N400 sites, that is, at centro-parietal sites, the strongest correlations were found at right frontocentral sites. These results should be considered with caution since, in contrast with centro-parietal locations, they do not correspond to a priori hypotheses. Nevertheless, they suggest that the amplitude of an ERP component other than the N400 could also depend on the severity of the disorganization trait. Future research should be devoted to assess the functional significance of this component. In any case, the N400 effect is known to be generated by multiple brain regions (Halgren, Dhond, Christensen, Van Petten, Marinkovic, et al., 2002), having multiple contributing electrophysiological sub-components (Franklin, Dien, Neely, Huber, \& Waterson, 2007). Disorganization may thus affect a sub-component of the N400 that is not maximal over centro-parietal electrodes.

As for the correlations observed at centro-parietal sites, given that a semantic categorization task was used, they are most likely due to a modulation of the classical N400 by the disorganization factor. This association of greater N400s with higher disorganization scores may have different functional significances depending on the hypothesis of reference. Regarding the hypothesis that N400 amplitudes index the automatic spreading activation within the lexicon (Deacon, Hewitt, Yang, \& Nagata, 2000; Kutas \& Hillyard, 1984), our results are consistent with studies using schizophrenia patients (Andrews et al., 1993; Salisbury et al., 2000), which suggest that schizophrenia, and more specifically thought disorders, may be related to an overspread activation in the network of word representations (Maher, Manschreck, Hoover, \& Weisstein, 1987; Manschreck, Maher, Milavetz, Ames, Weisstein, \& Schneyer, 1988; Spitzer, Braun, Hermle, \& Maier, 1993). This idea that words induce a broader activation in morethan in less-disorganized individuals implies that targets also induce broader activation. Therefore, according to the hypothesis that N400 is generated by the activation, all targets should elicit larger N400 deflections, and N400 effects may not change, as observed here. A similar interpretation could be made at the level of the network coding for meanings, following the hypothesis that N400 indexes activation in semantic memory (see Kutas \& Federmeier, 2000; Lau, Phillips, \& Poeppel, 2008). Alternatively, according to the hypothesis that the amplitude of the N400 is proportional to the efforts deployed by the brain to integrate the meaning of the stimulus in the context (Holcomb, 1993), our results indicate that healthy people with more disorganization scores deploy greater efforts to try to integrate target stimuli than people without disorganization. This may be a consequence of an initial overspread of activation since it arguably increases difficulty. However, the absence of effects of disorganization scores on error rates and reaction times does not provide further support to these lexical and semantic access views of N400s, because these processes are likely to be involved in the categorization task. Alternatively, according to a fourth hypothesis, the N400s index a late inhibition of inappropriate representations (Debruille, 1998; Debruille, Ramirez, Wolf, Schaefer, Nguyen, et al., 2008; for a review, see Debruille, 2007). In the case of the present experiment, these inappropriate representations could be those related to animals that were activated by the question word 'ANIMAL?' and that are inappropriate for non-exemplars targets. Due to their overspread of activation, subjects with higher disorganization scores would have activated more of these representations and thus would have more inhibition to perform, hence the greater N400 amplitudes. This hypothesis may also be used to interpret the greater N400s obtained in the exemplar 


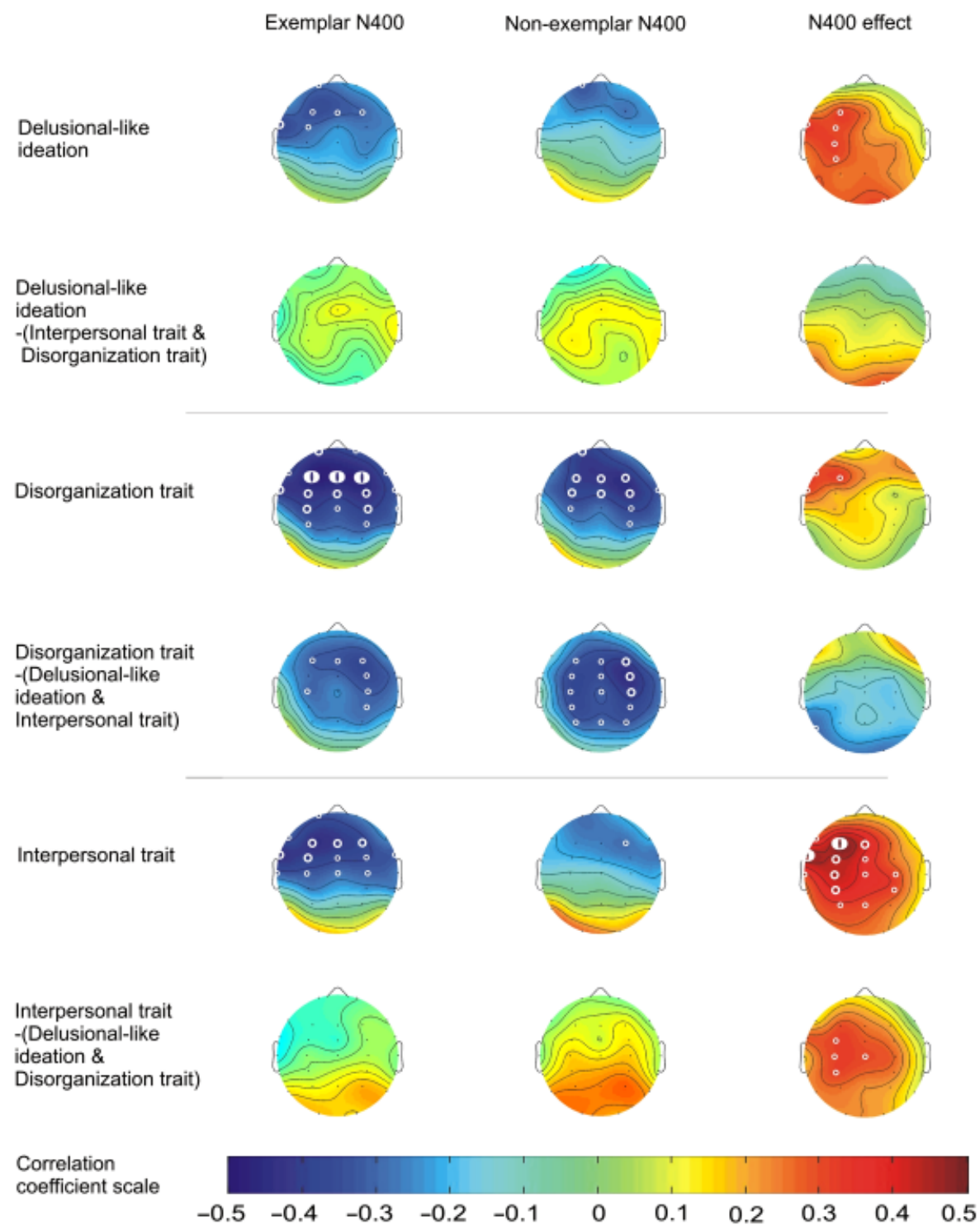

Figure 5. Spline interpolated maps of the correlation coefficients between mean ERP voltages in the 350-550 ms time window and schizotypal features. Bivariate correlations for each factor are followed by partial correlations that controlled for the two other factors' scores. The blue colors used for negative correlation coefficients mean that greater N400 amplitudes correlate to higher factor scores, as greater N400s (for exemplars, non-exemplars, and effect) are associated with more negative values. Significant correlations at one electrode site are tagged by a small ring for $p<.05$, by an intermediate ring for $p<.01$, and by a large ring for $p<.001$. No correction of the alpha level was necessary here despite the large number of statistical tests done because of the a priori hypothesis (i.e., that strongest correlation would be observed at centro-parietal sites, that is, at the sites where the N400 effect with written words is usually maximal). A Bonferroni correction taking into account all the tests done $(3$ traits $\times 28$ electrodes $=84)$ would set the alpha level at 0.0005 . With a less conservative correction, considering familywise errors, the alpha would be set at 0.0017 (for 28 electrodes).

condition. The first word 'ANIMAL' may activate knowledge corresponding to animals other than the target. This inappropriate activation may be more important in cases of more marked disorganization.

Another finding of the present study is that, in the N400 time window, the subtractions of the voltages of the exemplar category from those of the non-exemplar category positively correlated with higher scores for the interpersonal factor of the SPQ. The higher the score, the smaller the N400 effect, since this effect has a negative voltage. These results are consistent with those of a previous study (Kiang \& Kutas, 2005), which reported a correlation between ERP amplitude in the N400 time window and interpersonal scores in normals. Our study replicates this finding but also shows that it persists when controlling for the two re- maining schizotypal factors. Moreover, it suggests that these correlations are maximal over left centro-parietal electrodes (see Figure 5) whereas the typical sites where the N400 effect is maximal for written word stimuli are usually at the right centroparietal part of the scalp (Holcomb, 1988; Kutas et al., 1988). However, these results should be considered with caution since they do not correspond to a priori hypotheses. Nevertheless, they suggest that the correlations may be due to the modulations of an ERP other than the N400, the functional significance of which would have to be investigated. Alternatively, the interpersonal factor might preferentially affect a sub-component of the N400 that is not maximal over centro-parietal electrodes. This left centro-parietal preferential location is difficult to compare with findings in the literature. The correlations reported in other 
studies pertained to only one scalp location. In Kiang and Kutas (2005), it was the midline parietal electrode (Pz). In their more recent study in schizophrenia patients, Kiang et al. (2007) found that smaller N400 effects at the central electrode, $\mathrm{Cz}$, tend to correlate with the severity of negative symptoms, and thus with symptoms that resemble those measured by the SPQ interpersonal factor. Meanwhile, Kostova et al. (2005) found the same relationship between negative symptoms of patients and the $\mathrm{N} 400$ effect at $\mathrm{Pz}$, but this was only a trend $(p=.08)$. However, no measures of the correlations between the different clinical factors were made. It thus remains unclear whether it would have been necessary to control for other factors in their subjects' samples too, and whether the correlation with ERPs would persist with these controls.

The maps of the bivariate correlations obtained for the disorganization trait and those obtained for the interpersonal score are interesting to compare to the maps of partial correlations (Figure 5). This comparison reveals that controlling for the two other schizotypal factors may have dramatic effects. At some sites, this control induced a switch in the direction of the correlations. By emphasizing the importance of the control, these findings may help to reconcile some of the discrepancies present in the N400 studies of schizophrenia. These discrepancies could be due to varying patterns of symptom correlations across the population samples studied.

Regarding delusion-like ideation, the atypically localized correlations found between the severity of this factor and the amplitude of the ERPs in the N400 time window (Figure 5) disappeared when we controlled for the effect of the two other factors, unlike the correlations found in patients (Debruille et al.,
2007), which resisted this control. Therefore, it seems that delusion-like ideation may not be correlated to N400 in normals and that healthy subjects with delusion-like ideation do not have abnormal semantic processes. The present study thus suggests that delusion-like ideation observed in healthy subjects might not be similar to delusional beliefs of patients as they are not underlain by the same neurocognitive mechanisms. These results are at odds with the findings of Debruille et al. (2007) and Kiang et al. (2007) in schizophrenia patients and could be used to support a distinction between normal population and schizophrenia patients in contrast with the idea of a continuum between the two (Garety, Bebbington, Fowler, Freeman, \& Kuipers, 2007; Shevlin et al., 2007; Verdoux \& van Os, 2002). However, the results of Prévost, Rodier, Lionnet, Brodeur, King, and Debruille (forthcoming) suggest that smaller N400s can be found in healthy subjects with high scores on delusion-like ideation items of the SPQ, when boosting paranoid feelings, which probably diminishes some of the differences between schizophrenia patients and healthy subjects.

In summary, the results of the present study suggest that the semantic processes accompanying a particular schizotypal personality trait should be studied while controlling for the other traits. As with other brain imaging techniques, various localizations have to be examined to test whether the correlations actually pertain to the neurocognitive component under focus. Moreover, our study supports the view of a continuum between schizophrenia and normality at least for disorganization and interpersonal factors to the extent that they were found, in healthy subjects, to be accompanied by semantic processes biases similar to those observed in schizophrenia patients.

\section{REFERENCES}

American EEG Society. (1991). Electrode Nomenclature Committee. Modified expanded 10-20 system. Journal of Clinical Neurophysiology, 35, 200-202.

Andrews, S., Shelley, A. M., Ward, P. B., Fox, A., Catts, S. V., \& McConaghy, N. (1993). Event-related potential indices of semantic processing in schizophrenia. Biological Psychiatry, 34, 443-458.

Bedwell, J. S., Kamath, V., \& Compton, M. T. (2009). The relationship between interview-based schizotypal personality dimension scores and the continuous performance test. Schizophrenia Research, 108, 158-162.

Cadenhead, K., Kumar, C., \& Braff, D. (1996). Clinical and experimental characteristics of "hypothetically psychosis prone" college students. Journal of Psychiatric Research, 30, 331-340.

Chapman, L. J., Edell, W., \& Chapman, J. P. (1980). Physical anhedonia, perceptual aberration, and psychosis proneness. Schizophrenia Bulletin, 6, 639-653.

Claridge, G. (1997). Final remarks and future directions. In G. Claridge (Ed.), Schizotypy. Implications for illness and health (pp. 301-317). Oxford: Oxford University Press.

Cohen, J. D., \& Servan-Schreiber, D. (1992). Context, cortex, and dopamine: A connectionist approach to behavior and biology in schizophrenia. Psychological Review, 99, 45-77.

Coleman, M. J., Levy, D. L., Lenzenweger, M. F., \& Holzman, P. S. (1996). Thought disorder, perceptual aberrations, and schizotypy. Journal of Abnormal Psychology, 105, 469-473.

Content, A., Mousty, P., \& Radeau, M. (1990). Une base de données lexicales informatisée pour le français écrit et parlé. L'Année Psychologique, 90, 551-566.

Deacon, D., Hewitt, S., Yang, C., \& Nagata, M. (2000). Event-related potential indices of semantic priming using masked and unmasked words: Evidence that the N400 does not reflect a post-lexical process. Cognitive Brain Research, 9, 137-146.

Debruille, J. B. (1998). Knowledge inhibition and N400: A study with words that look like common words. Brain and Language, 62, 202220
Debruille, J. B. (2007). The N400 potential could index a semantic inhibition. Brain Research Review, 56, 472-427.

Debruille, J. B., Kumar, N., Saheb, D., Chintoh, A., Gharghi, D., Lionnet, C., et al. (2007). Delusions and processing of discrepant information: An event-related brain potential study. Schizophrenia Research, 89, 261-277.

Debruille, J. B., Kumar, N., Saheb, D., Chintoh, A., Gharghi, D., Lionnet, C., et al. (2010). Circumventing the deficit of context processing in schizophrenia: An event-related brain potential study. International Journal of Psychophysiology, 75, 167-176.

Debruille, J. B., Ramirez, D., Wolf, Y., Schaefer, A., Nguyen, T. V., Bacon, B. A., et al. (2008). Knowledge inhibition and N400: A within- and a between-subjects study with distractor words. Brain Research, 1187, 167-183.

Dickey, C. C., McCarley, R. W., Niznikiewicz, M. A., Voglmaier, M. M., Seidman, L. J., Kim, S., et al. (2005). Clinical, cognitive, and social characteristics of a sample of neuroleptic-naive persons with schizotypal personality disorder. Schizophrenia Research, 78, 297-308.

Ditman, T., \& Kuperberg, G. R. (2007). The time course of building discourse coherence in schizophrenia: An ERP investigation. Psychophysiology, 44, 991-1001.

Dumas, P., Bouafia, S., Gutknecht, C., Saoud, M., Dalery, J., \& d'Amato, T. (2000). [Validation of the French version of the Raine Schizotypal Personality Disorder Questionnaire - Categorial and dimensional approach to schizotypal personality traits in a normal student population]. Encephale, 26, 23-29.

Dumas, P., Rosenfeld, F., Saoud, M., Dalery, J., \& d'Amato, T. (1999). [Translation and French adaptation of the Raine Schizotypal Personality Questionnaire]. Encephale, 25, 315-322.

Franklin, M. S., Dien, J., Neely, J. H., Huber, E., \& Waterson, L. D. (2007). Semantic priming modulates the N400, N300, and N400RP. Clinical Neurophysiology, 118, 1053-1068.

Freeman, D. (2007). Suspicious minds: The psychology of persecutory delusions. Clinical Psychology Review, 27, 425-457. 
Garety, P. A., Bebbington, P., Fowler, D., Freeman, D., \& Kuipers, E. (2007). Implications for neurobiological research of cognitive models of psychosis: A theoretical paper. Psychological Medicine, 37, 13771391.

Gassab, L., Mechri, A., Dogui, M., Gaha, L., d'Amato, T., Dalery, J., et al. (2006). Abnormalities of auditory event-related potentials in students with high scores on the Schizotypal Personality Questionnaire. Psychiatry Research, 144, 117-122.

Greenhouse, G. W., \& Geisser, S. (1959). On methods of analysis of profile data. Psychometrika, 24, 1582-1589.

Gooding, D. C., Tallent, K. A., \& Hegyi, J. V. (2001). Cognitive slippage in schizotypic individuals. The Journal of Nervous and Mental Disease, 189, 750-756.

Halgren, E., Dhond, R. P., Christensen, N., Van Petten, C., Marinkovic, K., Lewine, J. D., et al. (2002). N400-like magnetoencephalography responses modulated by semantic context, word frequency, and lexical class in sentences. NeuroImage, 17, 1101-1116.

Hardy-Baylé, M. C., Sarfati, Y., \& Passerieux, C. (2003). The cognitive basis of disorganization symptomatology in schizophrenia and its clinical correlates: Toward a pathogenetic approach to disorganization. Schizophrenia Bulletin, 29, 459-471.

Hemsley, D. R. (2005). The schizophrenic experience: Taken out of context? Schizophrenia Bulletin, 31, 43-53.

Holcomb, P. J. (1988). Automatic and attentional processing: An eventrelated brain potential analysis of semantic priming. Brain and Language, 35, 66-85.

Holcomb, P. J. (1993). Semantic priming and stimulus degradation: Implications for the role of the N400 in language processing. Psychophysiology, 30, 47-61.

Johns, L. C., \& van Os, J. (2001). The continuity of psychotic experiences in the general population. Clinical Psychology Review, 21, 1125-1141.

Johnston, A. E., Rossell, S. L., \& Gleeson, J. F. (2008). Evidence of semantic processing abnormalities in schizotypy using an indirect semantic priming task. The Journal of Nervous and Mental Disease, 196, 694-701.

Kiang, M., \& Kutas, M. (2005). Association of schizotypy with semantic processing differences: An event-related brain potential study. Schizophrenia Research, 77, 329-342.

Kiang, M., Kutas, M., Light, G. A., \& Braff, D. L. (2007). Electrophysiological insights into conceptual disorganization in schizophrenia. Schizophrenia Research, 92, 225-236.

Kiang, M., Kutas, M., Light, G. A., \& Braff, D. L. (2008). An eventrelated brain potential study of direct and indirect semantic priming in schizophrenia. American Journal of Psychiatry, 165, 74-81.

Kostova, M., Passerieux, C., Laurent, J. P., \& Hardy-Baylé, M. C. (2005). N400 anomalies in schizophrenia are correlated with the severity of formal thought disorder. Schizophrenia Research, 78, 285291.

Kucera, H., \& Francis, W. N. (1967). Computational analysis of presentday American English. Providence, RI: Brown University Press.

Kuperberg, G. R., Sitnikova, T., Goff, D., \& Holcomb, P. J. (2006). Making sense of sentences in schizophrenia: Electrophysiological evidence for abnormal interactions between semantic and syntactic processing. Journal of Abnormal Psychology, 115, 251-265.

Kutas, M., \& Federmeier, K. D. (2000). Electrophysiology reveals semantic memory use in language comprehension. Trends in Cognitive Sciences, 4, 463-470.

Kutas, M., \& Hillyard, S. A. (1984). Brain potentials during reading reflect word expectancy and semantic association. Nature, 307, 161163.

Kutas, M., van Petten, C., \& Besson, M. (1988). Event-related potential asymmetries during the reading of sentences. Electroencephalography and Clinical Neurophysiology, 69, 218-233.

Lau, E. F., Phillips, C., \& Poeppel, D. (2008). A cortical network for semantics: (De)constructing the N400. Nature Review Neuroscience, 9, 920-933

Lenzenweger, M. F. (1999). Schizophrenia: Refining the phenotype, resolving endophenotypes. Behaviour Research and Therapy, 37, 281295.

Lenzenweger, M. F., \& Dworkin, R. H. (1996). The dimensions of schizophrenia phenomenology. Not one or two, at least three, perhaps four. British Journal of Psychiatry, 168, 432-440.

Maher, B. A., Manschreck, T. C., Hoover, T. M., \& Weisstein, C. C. (1987). Thought disorder and measured features of language pro- duction in schizophrenia. In P. Harvey \& E. Walker (Eds.), Positive and negative symptoms in psychosis: Description, research and future directions (pp. 195-215). Hillsdale, NJ: Erlbaum.

Manschreck, T. C., Maher, B. A., Milavetz, J. J., Ames, D., Weisstein, C. C., \& Schneyer, M. L. (1988). Semantic priming in thought disordered schizophrenic patients. Schizophrenia Research, 1, 61-66.

Moritz, S., \& Woodward, T. S. (2006). A generalized bias against disconfirmatory evidence in schizophrenia. Psychiatry Research, 142, $157-165$.

Peters, E. R., Joseph, S. A., \& Garety, P. A. (1999). Measurement of delusional ideation in the normal population: Introducing the PDI (Peters et al. Delusions Inventory). Schizophrenia Bulletin, 25, 553576

Prévost, M., Rodier, M., Lionnet, C., Brodeur, M., King, S., \& Debruille, J. B. Paranoid induction reduces N400s of healthy subjects with delusional-like ideation. Psychophysiology, forthcoming.

Raine, A. (1991). The SPQ: A scale for the assessment of schizotypal personality based on DSM-III-R criteria. Schizophrenia Bulletin, 17, 555-564.

Raine, A., Reynolds, C., Lencz, T., Scerbo, A., Triphon, N., \& Kim, D. (1994). Cognitive-perceptual, interpersonal, and disorganized features of schizotypal personality. Schizophrenia Bulletin, 20, 191-201.

Reynolds, C. A., Raine, A., Mellingen, K., Venables, P. H., \& Mednick, S. A. (2000). Three-factor model of schizotypal personality: Invariance across culture, gender, religious affiliation, family adversity, and psychopathology. Schizophrenia Bulletin, 26, 603-618.

Salisbury, D. F., O'Donnell, B. F., McCarley, R. W., Nestor, P. G., \& Shenton, M. E. (2000). Event-related potentials elicited during a context-free homograph task in normal versus schizophrenic subjects. Psychophysiology, 37, 456-463.

Schul, Y., Mayo, R., \& Burnstein, E. (2004). Encoding under trust and distrust: The spontaneous activation of incongruent cognitions. Journal of Personality and Social Psychology, 86, 668-679.

Shevlin, M., Murphy, J., Dorahy, M. J., \& Adamson, G. (2007). The distribution of positive psychosis-like symptoms in the population: A latent class analysis of the National Comorbidity Survey. Schizophrenia Research, 89, 101-109.

Sommer, I. E., Daalman, K., Rietkerk, T., Diederen, K. M., Bakker, S., Wijkstra, J., et al. (2008). Healthy individuals with auditory verbal hallucinations; Who are they? Psychiatric assessments of a selected sample of 103 subjects. Schizophrenia Bulletin, in press.

Spitzer, M., Braun, U., Hermle, L., \& Maier, S. (1993). Associative semantic network dysfunction in thought-disordered schizophrenic patients: Direct evidence from indirect semantic priming. Biological Psychiatry, 34, 864-877.

Strauss, J. S. (1969). Hallucinations and delusions as points on continua function. Rating scale evidence. Archives of General Psychiatry, 21, 581-586.

van Os, J., Hanssen, M., Bijl, R. V., \& Ravelli, A. (2000). Strauss (1969) revisited: A psychosis continuum in the general population? Schizophrenia Research, 45, 11-20.

Verdoux, H., \& van Os, J. (2002). Psychotic symptoms in non-clinical populations and the continuum of pychosis. Schizophrenia Research, 54, 59-65.

Wan, L., Crawford, H. J., \& Boutros, N. (2006). P50 sensory gating: Impact of high vs. low schizotypal personality and smoking status. International Journal of Psychophysiology, 60, 1-9.

Wang, J., Miyazato, H., Hokama, H., Hiramatsu, K., \& Kondo, T. (2004). Correlation between P50 suppression and psychometric schizotypy among non-clinical Japanese subjects. International Journal of Psychophysiology, 52, 147-157.

Wilson, C. M., Christensen, B. K., King, J. P., Li, Q., \& Zelazo, P. D. (2008). Decomposing perseverative errors among undergraduates scoring high on the Schizotypal Personality Questionnaire. Schizophrenia Research, 106, 3-12.

Woodward, T. S., Buchy, L., Moritz, S., \& Liotti, M. (2007). A bias against disconfirmatory evidence is associated with delusion proneness in a nonclinical sample. Schizophrenia Bulletin, 33, 1023-1028.

(ReCEIVED January 31, 2009; ACCEPTED November 21, 2009) 